Article

\title{
Monitoring Changes in Rice Cultivated Area from SAR and Optical Satellite Images in Ben Tre and Tra Vinh Provinces in Mekong Delta, Vietnam
}

\author{
Kirsi Karila *, Olli Nevalainen, Anssi Krooks, Mika Karjalainen and Sanna Kaasalainen \\ Finnish Geodetic Institute, Geodeetinrinne 2, FI-02431 Masala, Finland; \\ E-Mails: olli.nevalainen@fgi.fi (O.N.); anssi.krooks@fgi.fi (A.K.); \\ mika.karjalainen@fgi.fi (M.K.); sanna.kaasalainen@fgi.fi (S.K.) \\ * Author to whom correspondence should be addressed; E-Mail: Kirsi.Karila@fgi.fi; \\ Tel.: +358-295-308-034; Fax: +358-929-555-211.
}

Received: 13 January 2014; in revised form: 8 April 2014 / Accepted: 24 April 2014 /

Published: 2 May 2014

\begin{abstract}
The objective of this study was to obtain up-to-date information on land use and to identify long term changes in land use, especially rice, aquaculture and other crops in Ben Tre and Tra Vinh provinces in Vietnam's Mekong Delta. Long-term changes in land-use of the study area have not been studied using long time series of SAR and optical Earth observation (EO) data before. EO data from 1979-2012 was used: ENVISAT ASAR Wide Swath Mode, SPOT and Landsat imagery. An unsupervised ISODATA classification was performed on multitemporal SAR images. The results were validated using ground truth data. Using the Synthetic Aperture Radar (SAR) imagery maps for 2005, 2009 and 2011 were obtained. Different rice crops, aquaculture and fruit trees could be distinguished with an overall accuracy of $80 \%$. Using available optical imagery the time series was extended from 2005 to 1979 . Long-term decrease in the rice acreage and increase in the aquaculture acreage could be detected.
\end{abstract}

Keywords: land-use change; Mekong Delta; Envisat ASAR WSM; Landsat; SPOT; multitemporal SAR; ground truth data; rice; aquaculture 


\section{Introduction}

Rice is one of the most important nutrition components in the world and a supply of stable food in many countries, especially in Southeast Asia. Up-to-date information on areas under rice cultivation and changes in the cultivated area is essential for national political decision-makers and for international trade organizations for planning and monitoring purposes. Due to the human population growth the global food security has to be ensured. Irrigated rice fields are also among the major sources of methane emissions. On the other hand, awareness of changes in rice growing conditions is beneficial for estimating the long term impacts of climate change on the rice production.

According to the International Rice Research Institute, Vietnam is the leading producer of rice in the world; the Mekong Delta region being the most important rice production area [1]. In some of the coastal parts of the Mekong Delta region, United Nations International Fund for Agriculture (IFAD) has given recommendations to rice farmers to change from rice to other crops due to the salinity intrusion in the area. Therefore, information on areas under aquaculture and other crops is needed as well. Land-use changes can be further used to evaluate the effect of salinity intrusion to land use in the study area and to define the rice growing areas that need to transit to other land uses as a result of increasing salinity currently and through forecasting based on the sea level rise and reduced river flow. Although, some central descriptive data on cultivated areas are recorded by the national authorities, there is still need for more accurate and timely information. Moreover, it would be advantageous to extend the time-line of changes in rice land-use to the past decades, starting even from the 1970s. The use of Earth Observation (EO) satellites and their historical data archives is a reliable and cost effective way to obtain this information.

The main challenge in estimating the area under rice cultivation using EO data is to obtain well-timed satellite data, which enables interpretation of rice areas from other crops and land-use classes. Therefore, a multi-temporal data set is typically needed, i.e., a sufficient number of images during the rice growing season (there may be one to even three growing seasons in a year). Due to the extensive cloud cover during the rice growing season, the use of cloud-penetrating imaging system, Synthetic Aperture Radar (SAR) on-board of satellite, appears to be an ideal solution for monitoring of agricultural areas [2]. Using a priori knowledge of common cultivation practices in the target area (crop calendar), it is possible to order well-timed SAR images for detecting rice areas. It is also advantageous to exploit optical EO data to identify areas that are permanently non-irrigated, since there are different interactions with the target for active microwave SAR and daylight-based optical sensors. In addition, optical satellite missions have been operational longer than SAR satellites and have collected long time series of image data. Examples of integrated use of SAR and optical data for land cover and crop type mapping are presented in [3-16].

A review of remote sensing of rice crop areas has been published [17]. In Mekong Delta, several land cover studies based on optical EO data have been carried out (e.g., [18-21]). The German-Vietnamese WISDOM (Water-related Information System for the sustainable Development of the Mekong Delta in Vietnam) project is currently designing and implementing an information system for the Mekong Delta, containing information from the fields of hydrology, sociology, information technology and earth observation [22]. A considerable amount of scientific research has been carried on the matter of rice crop and land cover monitoring using SAR data, e.g., [2,23-30]. These results are promising, 
providing users with accurate information on the rice cultivated areas and even the estimation of potential rice yields was demonstrated. These earlier demonstrations were based on ERS-1, ERS-2, Radarsat-1, and Envisat SAR data, but these satellites are not operational any longer. At the moment, possible SAR data sources are TerraSAR-X/TanDEM-X, COSMO-SkyMed, and Radarsat-2. The satellite constellations of several satellites enable shorter revisit times and more frequent monitoring of agricultural areas. However, considering the operational rice crop monitoring an important data source in the future is the Sentinel-1 SAR satellites (ESA), which is a continuation to the C-band ERS and Envisat SAR data series. ESA's free-of-charge data policy is also an obvious advantage of the Sentinel-1 system compared to other SAR satellite data. Therefore, this study exploits the verified, well-known, ESA Envisat C-band SAR data in crop type monitoring.

The objective of this study is to obtain up-to-date land-use information and identify long term trends in changes in land use in Tra Vinh and Ben Tre provinces, which are the main interest areas to IFAD in the Mekong Delta region. The specific interest was put on the rice cultivated areas due to the salinity intrusion in the area; however, other crops (sugarcane, coconut, and orchard) and aquaculture were also included in the study. The hypothesis was that this information can be obtained using historical EO data and a combination of all available radar and optical images. The goal was to use standard automatic image classification procedures and validate the results using ground truth data and historical changes based on interviews with local farmers. The contribution of this study is that it combines SAR and optical imagery into a long-term time series of land use change. This information has been sparsely available in the area.

\section{Materials and Methods}

\subsection{Study Area}

Ben Tre and Tra Vinh provinces (Figure 1) are located on the coast of the South China Sea in Vietnam. The area of interest is $8500 \mathrm{~km}^{2}$ and centered in $106.4^{\circ} \mathrm{E}, 9.9^{\circ} \mathrm{N}$ (UTM 48 WGS84). Terrain height is only few meters above sea level. The land use mainly consists of rice, orchard, coconut, sugar cane, aquaculture and urban and rural settlements. The rainy season lasts from May to October/November and the dry season from November/December to April. The distribution of rainfall is unequal increasing from south-west to north-east [31].

In the Mekong Delta region, Vietnam, salinity intrusion occurs in ocean coastal areas, where saline water moves into freshwater aquifers. This can lead to contamination of drinking water sources and problems in agriculture. The salinity intrusion happens naturally to some extent, however, climate change and changes in rainfall are causing more severe salinity intrusion. In addition to the salinity intrusion, drought and rising temperatures cause problems to agriculture and aquaculture. The salinity intrusion may be affected by the climate change, e.g., changes in rainfall, sea level rise and storms.

During the dry season salinity becomes a problem in the coastal areas. Even though surface water is very abundant, over three quarters of the Ben Tre surface water areas (water in rivers, canals, lowland areas) are affected by salinity from two or three months to the whole year [32]. The salinity intrusion is constrained to some extent using dykes, sluices and sand dunes along the coast. 
In general, when farmers rely on wet season rainfall, the salinity intrusion results in situation where there is sufficient time to grow only one rice crop per year. Farmers may also use fresh water from network of canals to provide supplementary irrigation where required, although in years when saline water intrusion occurs early, this is not possible. Over the past 30-40 years, many rice farmers in the saline affected areas have adapted to the natural conditions by growing rice in the wet season, then using the rice fields for growing shrimp (aquaculture) in the dry season [33].

Figure 1. A district map of the study area with the ground truth point (GTP) locations.

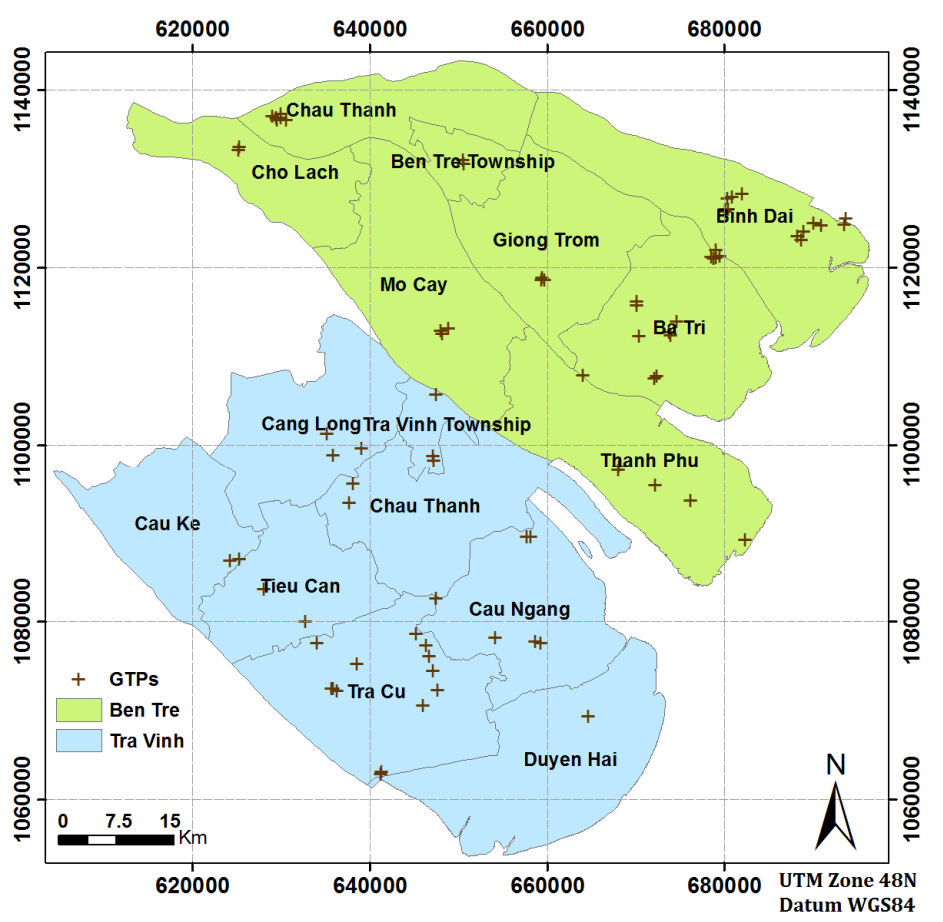

In the recent years in Ben Tre, the ratio of fruit trees output value has grown sharply. In 2002, the cultivation scheme was moving toward the reduction of low-yield rice fields, switching to aquaculture, sugar cane crops and fruit trees [34]. In Tra Vinh, rice is still the most important crop.

\subsection{Data Sets}

Long-term changes in land use in the Ben Tre and Tra Vinh provinces were monitored using EO data. A long time series of satellite data with medium resolution was required in order to cover the target area. According to a careful inspection of EO data archives, historical images were available from the SPOT, Landsat and ENVISAT satellites. The ENVISAT ASAR images were used to detect changes during 2005-2011. One to three rice crops per year are harvested in the area [18]. Therefore, several SAR images acquired throughout the year were needed for each year in order to map rice areas and to get the number of rice crops. Long term land-use changes from 1979 were monitored by the optical Landsat images and from 1987 using the optical SPOT images. The long term changes were detected from optical images acquired at the same time of year in December to January.

The following EO data (Tables 1 and 2) were acquired from European Space Agency (ESA) and United States Geological Survey (USGS) archives: 
- 38 ENVISAT ASAR Wide Swath Mode medium resolution images-2005, 2009, 2011

- Two Landsat images - 1979 and 2002

- $\quad$ Six SPOT images-1987, 1998, 2005

In order to get more data, in the 2011 SAR dataset, imagery from the end of 2010 and the beginning of 2012 were included. The pixel size of the SAR data is $75 \mathrm{~m}$. The Landsat image resolution is $80 \mathrm{~m}$ for the MSS image and $30 \mathrm{~m}$ for the ETM image. The resolution of the SPOT multispectral images was $20 \mathrm{~m}$. It should be noted that SPOT images did not cover the entire area of interest; however, with two images it was possible to cover $80 \%-90 \%$ of the area.

Table 1. The list of ENVISAT ASAR WSM images. Images are grouped into multitemporal sets: 2005 (1-13), 2009 (14-28) and 2011 (29-37).

\begin{tabular}{cccccccccccc}
\hline ID & Date & Orbit & Track & ID & Date & Orbit & Track & ID & Date & Orbit & Track \\
\hline 1 & 16.12 .2004 & 14,615 & 32 & 14 & 10.1 .2009 & 35,886 & 261 & 29 & 15.12 .2010 & 45,966 & 262 \\
2 & 19.12 .2004 & 14,658 & 75 & 15 & 6.4 .2009 & 37,117 & 490 & 30 & 3.1 .2011 & 46,239 & 104 \\
3 & 1.1 .2005 & 14,844 & 261 & 16 & 11.5 .2009 & 37,618 & 490 & 31 & 14.1 .2011 & 46,397 & 262 \\
4 & 12.3 .2005 & 15,846 & 261 & 17 & 17.5 .2009 & 37,704 & 75 & 32 & 2.2 .2011 & 46,670 & 104 \\
5 & 8.5 .2005 & 16,662 & 75 & 18 & 2.6 .2009 & 37,933 & 304 & 33 & 4.3 .2011 & 47,101 & 104 \\
6 & 6.6 .2005 & 16,390 & 304 & 19 & 9.6 .2009 & 38,041 & 412 & 34 & 15.3 .2011 & 47,259 & 262 \\
7 & 30.7 .2005 & 17,850 & 261 & 20 & 18.6 .2009 & 38,162 & 32 & 35 & 26.3 .2011 & 47,417 & 420 \\
8 & 2.8 .2005 & 17,893 & 304 & 21 & 4.7 .2009 & 38,391 & 261 & 36 & 3.4 .2011 & 47,532 & 104 \\
9 & 18.8 .2005 & 18,122 & 32 & 22 & 20.7 .2009 & 38,620 & 490 & 37 & 28.3 .2012 & 52,704 & 104 \\
10 & 8.10 .2005 & 18,852 & 261 & 23 & 24.8 .2009 & 39,121 & 490 & & & & \\
11 & 11.10 .2005 & 18,895 & 304 & 24 & 27.8 .2009 & 39,164 & 32 & & & & \\
12 & 1.12 .2005 & 19,625 & 32 & 25 & 1.10 .2009 & 39,665 & 32 & & & & \\
13 & 4.12 .2005 & 19,668 & 75 & 26 & 10.12 .2009 & 40,667 & 32 & & & & \\
& & & & 27 & 13.12 .2009 & 40,710 & 75 & & & & \\
& & & & 28 & 26.12 .2009 & 40,896 & 261 & & & & \\
\hline
\end{tabular}

Table 2. The list of optical satellite images.

\begin{tabular}{cccc}
\hline Satellite Instrument & Date & Path & Row \\
\hline Landsat 3 MSS & 25.1 .1979 & 134 & 53 \\
Landsat 7 ETM & 14.12 .2002 & 125 & 53 \\
Spot1 HRV & 23.12 .1987 & 276 & 330 \\
Spot1 HRV & 23.12 .1987 & 276 & 331 \\
Spot1 HRV & 3.1 .1998 & 276 & 330 \\
Spot1 HRV & 3.1 .1998 & 276 & 331 \\
Spot4 HRVIR & 18.1 .2005 & 276 & 330 \\
Spot4 HRVIR & 18.1 .2005 & 276 & 331 \\
\hline
\end{tabular}

In addition to the EO data, administrative boundaries, the official land-use map 2005 of the Mekong Delta from National Institute of Agricultural Planning and Projection (NIAPP) [18], scanned topographic maps from USGS [35] and the 3 arc-second digital elevation model (DEM) from the Shuttle Radar Topography Mission (SRTM) were used. The USGS topographic maps were geocoded and transformed to UTM48 WGS84 coordinate system. 
Ground truth data were acquired by a field survey campaign on 7-10 October 2013 in Ben Tre and Tra Vinh provinces. At first, 180 ground truth points were randomly created within the study area. However, ground truth data collection was laborious because all locations need to be visited including the time reserved for discussions with local people. The final number and spatial distribution of ground truth points were limited due to resources, time and road accessibility and 75 points were finally collected (Figure 1). The 75 ground truth points covered all land-use classes included in the classification maps. A higher number of points would be needed for detailed accuracy assessment of each land-use class. However, 75 points was considered to be enough for a rough estimate of the classification accuracy. In addition, farmers were asked about the past changes in land use. The number of various land-use classes included in field survey points is presented in Table 3. Detailed information about the crop cycle and type of each location was acquired by interviewing local authorities and farmers in situ. The accurate location of each field survey point was captured using hand-held Garmin Etrex GNSS navigator with location accuracy less than $10 \mathrm{~m}$.

Table 3. Summary of the ground truth points.

\begin{tabular}{cc}
\hline Land-Use Class & Number of Ground Truth Points \\
\hline Water & 4 \\
$2 \times$ Irrigated rice crop & 2 \\
Aquaculture & 20 \\
$2 \times$ Rainfed rice crop & 1 \\
Rainy season rice and shrimp culture & 4 \\
Settled areas, coconut, sugar cane and orchard & 19 \\
$3 \times$ Irrigated rice crop & 21 \\
Urban & 4 \\
\hline
\end{tabular}

\subsection{Methods}

\subsubsection{Image Data Preprocessing}

The SAR data consisted of 37 ENVISAT ASAR WSM (Wide-Swath Mode) images acquired between December 2004 and March 2012 (Table 1). All the SAR images were HH-polarized and from descending pass orbits. The specific product type of the images is ENVISAT ASAR WSM 1P. NEST (Next ESA SAR Toolbox) and ERDAS IMAGINE were used to perform the required pre-processing steps. Standard SAR processing methods were used for the ENVISAT ASAR WSM data. The SAR image processing included applying DORIS (The Doppler Orbitography and Radio-positioning Integrated by Satellite) precise orbit state vectors generated by the Centre de Traitement Doris Poseidon (CTDP) and radiometric correction using latest the ENVISAT auxiliary files. Thereafter, orthorectification by Range-Doppler terrain correction was carried out using the SRTM DEM (3 arc-second grid) and bilinear interpolation as the image and DEM resampling method. In addition, to enhance the image quality, Frost speckle suppression [36] with a window size of $3 \times 3$ pixels was applied. The Frost filter is a standard tool for SAR speckle suppression and the window size was selected to avoid degradation of spatial resolution [37]. Finally, the ENVISAT images were stacked to three multichannel images (multitemporal multiband image). 
The eight optical satellite images from Landsat and Spot programs were used for the land-use classification of Ben Tre and Tra Vinh provinces for years 1979, 1987, 1995, 2002, and 2005 (Table 2). The ERDAS IMAGINE 2013 software package was used for the optical image processing. In the orthorectification process (using the topographic maps and SRTM DEM), clearly visible point-type features with known map coordinates, such as road junctions and bridges, were used. The estimated accuracy of the orthorectified products were in order of 0.6-0.8 pixels. The DEM resolution was not a problem since the area is relatively flat. Moreover, to enhance the radiometric image quality for the classification purposes, a standard atmospheric correction and color adjustment were carried out (de-haze [38] and color stretch) using the ERDAS IMAGINE 2013 software. In addition, the SPOT images were mosaicked since each SPOT scene consists of two consecutive frames.

\subsubsection{Land-Use Classification}

Because the aim was to study trends in the land-use changes and not to develop new methodologies for image classification, standard image classification procedures were used. In order to get comparable results for change detection, an unsupervised classification method was selected. ISODATA (Iterative Self-Organizing Data Analysis Technique) [39] is a commonly used unsupervised classifier. Basically, it is an iterative clustering method which assigns pixels to clusters (classes) based on class means and a minimum distance rule. The clustering is continued until the maximum number of iterations has been performed or a maximum percentage of changed pixels has been reached between two iterations. The ISODATA algorithm is widely used in land-use classification and several scientific studies have been carried out using ISODATA clustering for EO data e.g., [18,19,40].

After the preliminary processing steps, the image stacks were classified using the unsupervised ISODATA classifier. The image stacks were classified to 40 clusters using 10 iterations and convergence threshold of 0.95 . The total number of clusters was varied systemically and 40 clusters were considered enough to recognize all the relevant land-use classes in the study area that could be distinguished with the used SAR data. Similarly, for the optical data 36 clusters were applied. After the ISODATA classification steps, the clusters were manually merged into eight (SAR) or seven (optical) final land-use classes. Urban areas and non-cultivated agriculture (i.e., rice field after harvesting) are easily mixed in optical data. Therefore, urban class was not used for optical data. The official land-use map 2005 of NIAPP [18] was used as a reference map to visually recognize what land-use class each of the clusters represented. In addition, the SPOT optical data was used for visual interpretation. Due to the lack of reference data, the derived land-use map of 2005 was used as a reference to merge the classes of the classified 2009 image stack and the derived land-use map of 2009 was again used as a reference for merging the 2011 classes. Settled areas, coconut, sugarcane and orchard were classified into the same class, because of the low data resolution. The final classes were:

1. Water

2. $2 \times$ Irrigated rice crop

3. Aquaculture

4. $2 \times$ Rainfed rice crop

5. Rainy season rice and shrimp culture (Rice in the rainy season and shrimp in the dry season)

6. Settled areas, coconut, sugar cane and orchard 
7. $3 \times$ Irrigated rice crop

8. Urban (only SAR)

The summary of the land-use maps produced is presented in Tables 4 and 5 .

Table 4. Summary of the land-use maps derived from ENVISAT ASAR images.

\begin{tabular}{cccc}
\hline & ENVISAT Map 2005 & ENVISAT Map 2009 & ENVISAT Map 2011 \\
\hline Number of images & 13 & 15 & 9 \\
Date begin & 16.12 .2004 & 10.1 .2009 & 15.12 .2010 \\
Date end & 4.12 .2005 & 26.12 .2009 & 28.3 .2012 \\
Pixel size (m) & & 75 & \\
\hline
\end{tabular}

Table 5. Summary of the land-use maps derived from SPOT and Landsat optical imagery.

\begin{tabular}{ccc}
\hline & SPOT Maps & Landsat Maps \\
\hline Satellite instrument & SPOT 1,3 and 4 & Landsat 3 (MSS) and 7 (ETM) \\
Channels & 4 & 4 (MSS), 7 (ETM) \\
Pixel size & $20 \mathrm{~m}$ & $60 \mathrm{~m}(\mathrm{MSS}), 15 \mathrm{~m}(\mathrm{ETM})$ \\
Maps for year & $1987,1998,2005$ & 1979,2002 \\
\hline
\end{tabular}

\subsubsection{Accuracy Assessment}

To validate the observations, we complemented the EO observations with a field survey campaign, where ground truth points were collected and interviews of the locals were carried out to validate the results. By including the interviews, we could improve the reliability of the results obtained from satellite images acquired before 2005.

The classification of the different crop cycles requires EO imagery, which is distributed evenly throughout the year and covers all crop cycles, so that the changes from one crop to another can be clearly detected. However, this was not the case for every input image set. In addition, the collected ground truth points included mostly " $3 \times$ irrigated rice crops" and not enough " $2 \times$ irrigated and $2 \times$ rainfed rice crops" to properly validate classification of various rice crop types. Thus, rice crop classes " $2 \times$ Irrigated rice crop", " $2 \times$ Rainfed rice crop" and " $3 \times$ Irrigated rice crop" were merged into one rice class for validation. In addition, the "Rainy season rice and shrimp culture" class was merged with the "Aquaculture" class resulting in the "Aquaculture2" class for SPOT 2005 land-use map validation, because the 2005 SPOT image was captured during the dry season when the "Rainy season rice and shrimp culture" areas were used for shrimp farming.

\section{Results and Discussion}

\subsection{Land-Use Change Results}

The resulting land-use maps from the ENVISAT data are presented in Figure 2. As expected, the most reliable results were based on SAR imagery, the reason being that the SAR data was multitemporal covering the whole year. As an instrument, SAR is sensitive to surface roughness and thus water surfaces such as rice paddies and aquaculture are easily detected. The multitemporal information can be used to separate the classes from each other. 
Figure 2. The land-use classifications from ENVISAT ASAR imagery
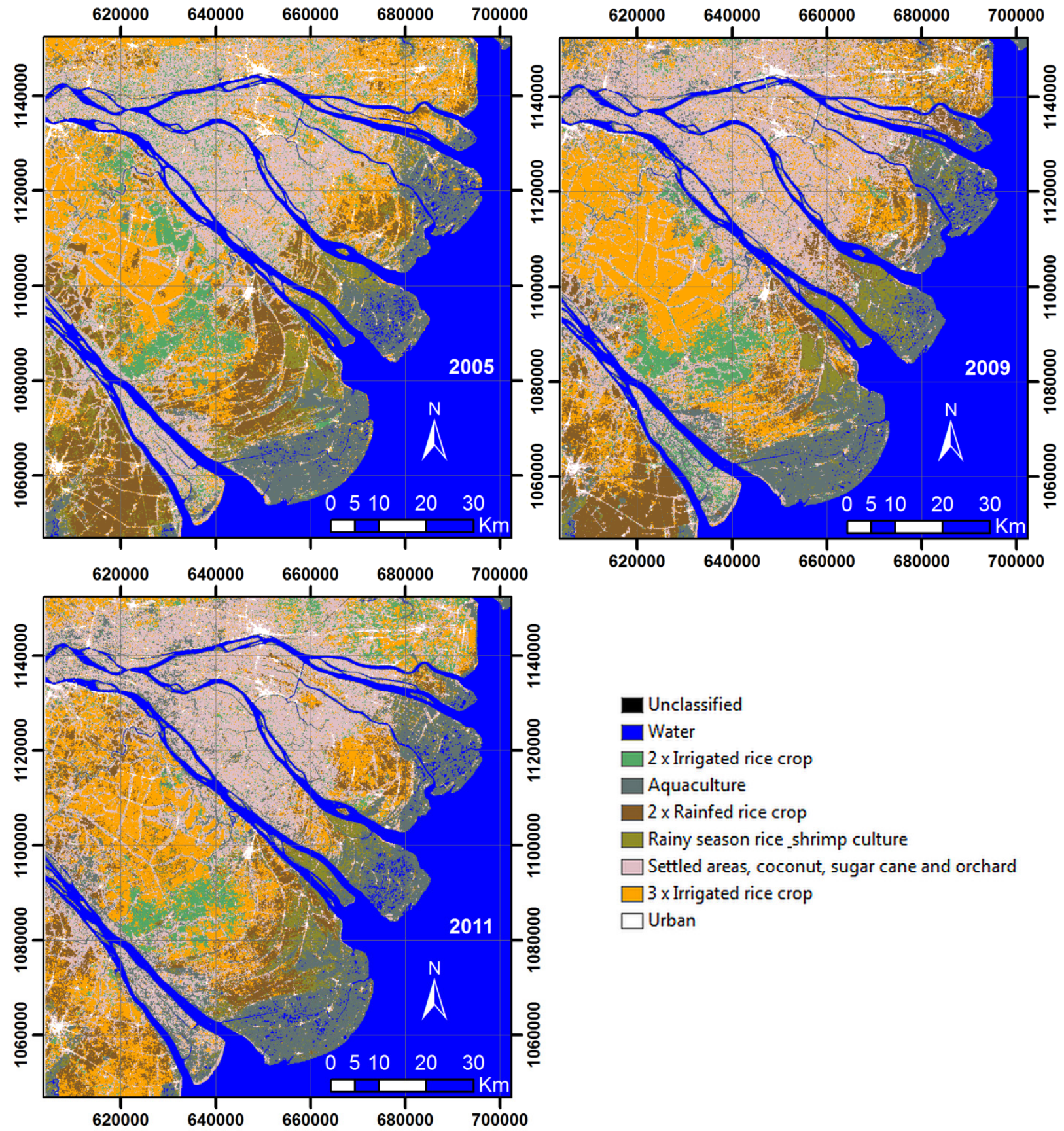

The land-use maps from SPOT are presented in Figure 3. The results from optical EO data were highly influenced by the dates of the images since the classification was based on a single image only. To get more reliable results, multitemporal (within one year) optical data would be required; however, due to the cloudiness in the area it was impossible to acquire such a data set. In addition, the spatial extent of the SPOT images was limited, which caused missing data in some districts, especially in Ben Tre. The farming practices (number of rice crops per year) might also have changed dramatically in the area when high yielding rice varieties have been adopted [41], and it is possible that the class division was not suitable for the 1979 Landsat image. 
Figure 3. The land-use classifications from the SPOT imagery.

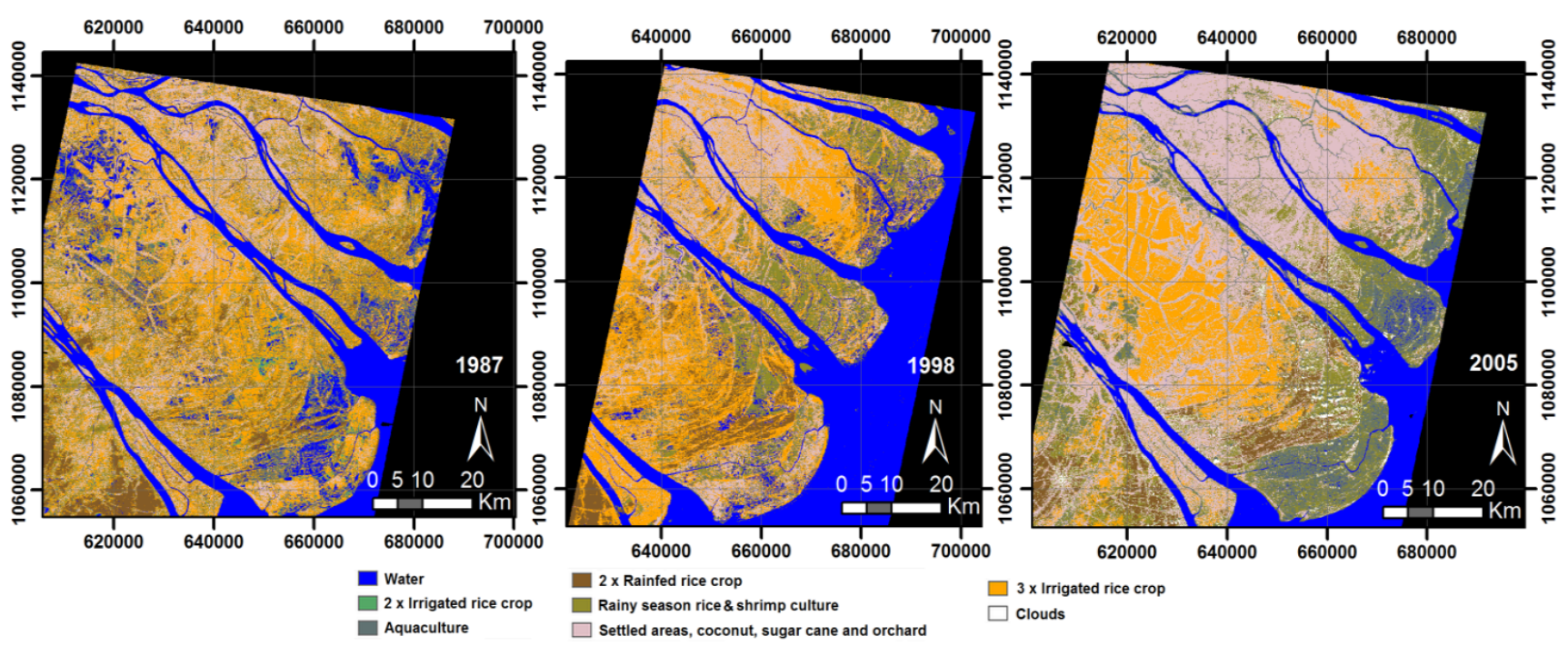

Even though the SAR data was based on multitemporal imagery, the image acquisition dates were not the most optimally distributed throughout the years 2005 and 2011. For the purposes of reliable statistical data analysis, we combined three rice classes to a single class, hereafter: "All rice", being the sum of " $2 \times$ irrigated", " $2 \times$ rainfed" and " $3 \times$ irrigated rice" crop classes in order to decrease the influence of variable image acquisition dates within a year. In order to analyze the optical imagery results, "Rainy season shrimp and rice" and "Aquaculture" were also combined to the "Aquaculture2" class. The Landsat results using the combined classes are presented in Figure 4.

Figure 4. The land-use classifications from the Landsat imagery.

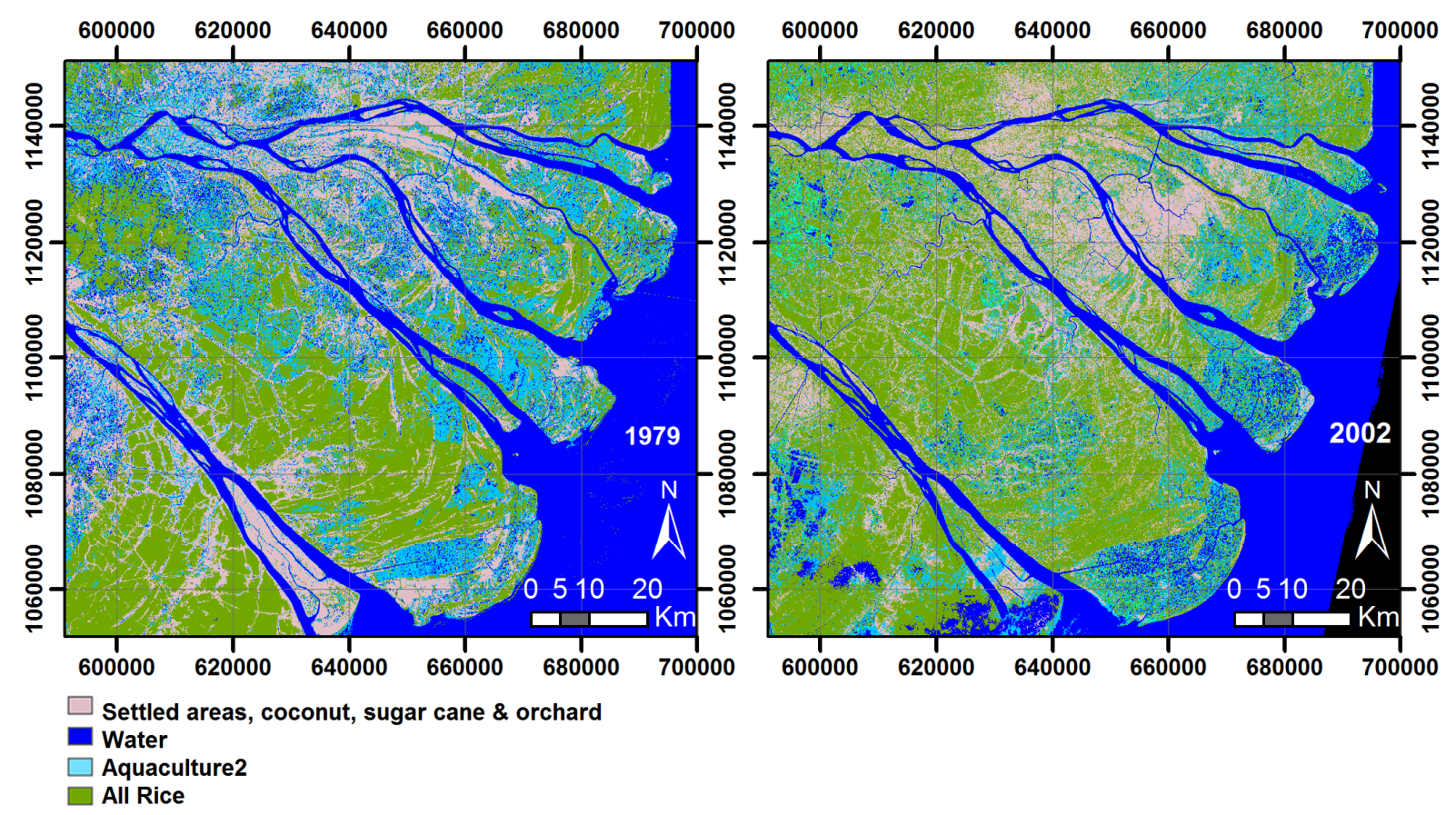

Thematic maps were produced from the classification result based on the SAR data for the classes "All rice" and "Aquaculture" (Figure 5). The change in hectares in comparison to the total area of a district was calculated and the map was colored accordingly. It can be noted that the most significant 
changes for rice have taken place in the inland districts. According to our experience during the field survey, it is highly possible that land-use changes in the coastal districts had already taken place before the year 2005 .

Figure 5. The relative change (\%) in acreage of rice cultivation (Left) and aquaculture (Right) based on the SAR classification results. The red color denotes the decrease in acreage and green denotes the increase in acreage.
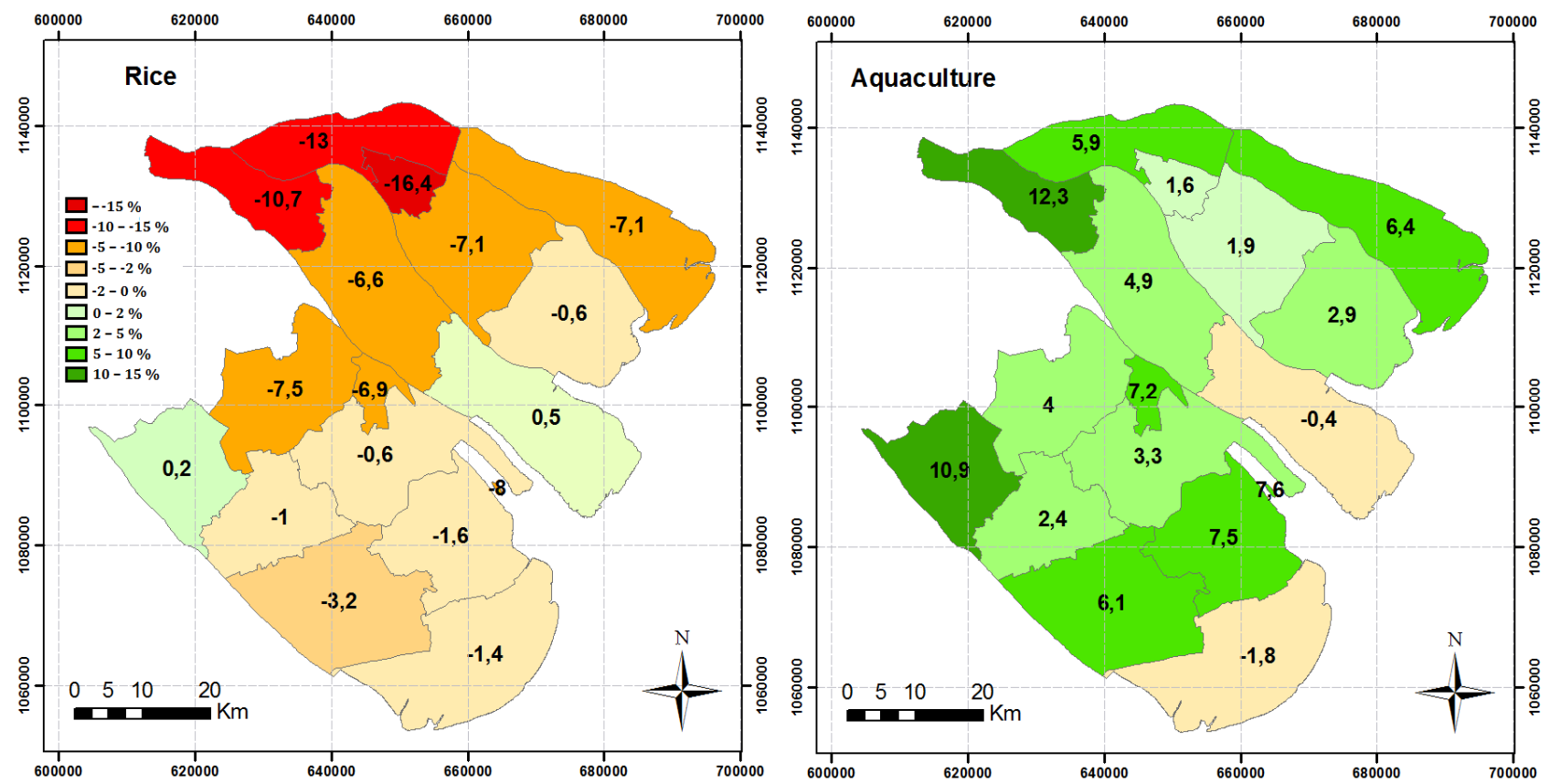

More detailed results on the land-use changes are presented for Ben Tre and Tra Vinh provinces in Figure 6. The 2011 SAR image set was lacking input data in May to December, which may have affected the detection of certain types of rice and the shrimp/rice fields. In Ben Tre, the SAR images show a decrease in the total rice acreage and increase in the aquaculture acreage. The acreage for "Settled areas, coconut, sugar cane and orchard" has increased as well. In the Tra Vinh province, the SAR images show a decrease in the rice acreage and an increase in the aquaculture acreage as well. The classes "Water" and "Urban" did not change remarkably between 2005 and 2011.

The land-use situation in 1979, 1987, 2002 and 2005, based on the Landsat and SPOT images, is presented in Figure 7. The 1998 SPOT result is not listed as the data is missing for some districts due to the image extent. The results show some decrease in the class "all rice" for 2005; however, the trend appears not to be very clear. The different imaging systems of the Landsat and SPOT satellites and different farming practices in the older data may also affect these results. Some water surfaces were classified into the "Water" class even if they actually belonged to the aquaculture areas, because the single-date images cannot distinguish these classes. Also, floods in the area may affect the results when water is covering other land-uses.

In Figure 8, the SPOT results $(1987,1998,2005)$ for four districts in Tra Vinh are presented. Duyen Hai is a coastal district and aquaculture has clearly increased there as rice acreage has decreased. The trend is notable in the other three districts as well. 
Figure 6. The total acreages of land-use classes in Ben Tre and Tra Vinh provinces based SAR images for 2005, 2009 and 2011. "All rice" is the sum of $2 \times$ irrigated, $2 \times$ rainfed and $3 \times$ irrigated rice crop.

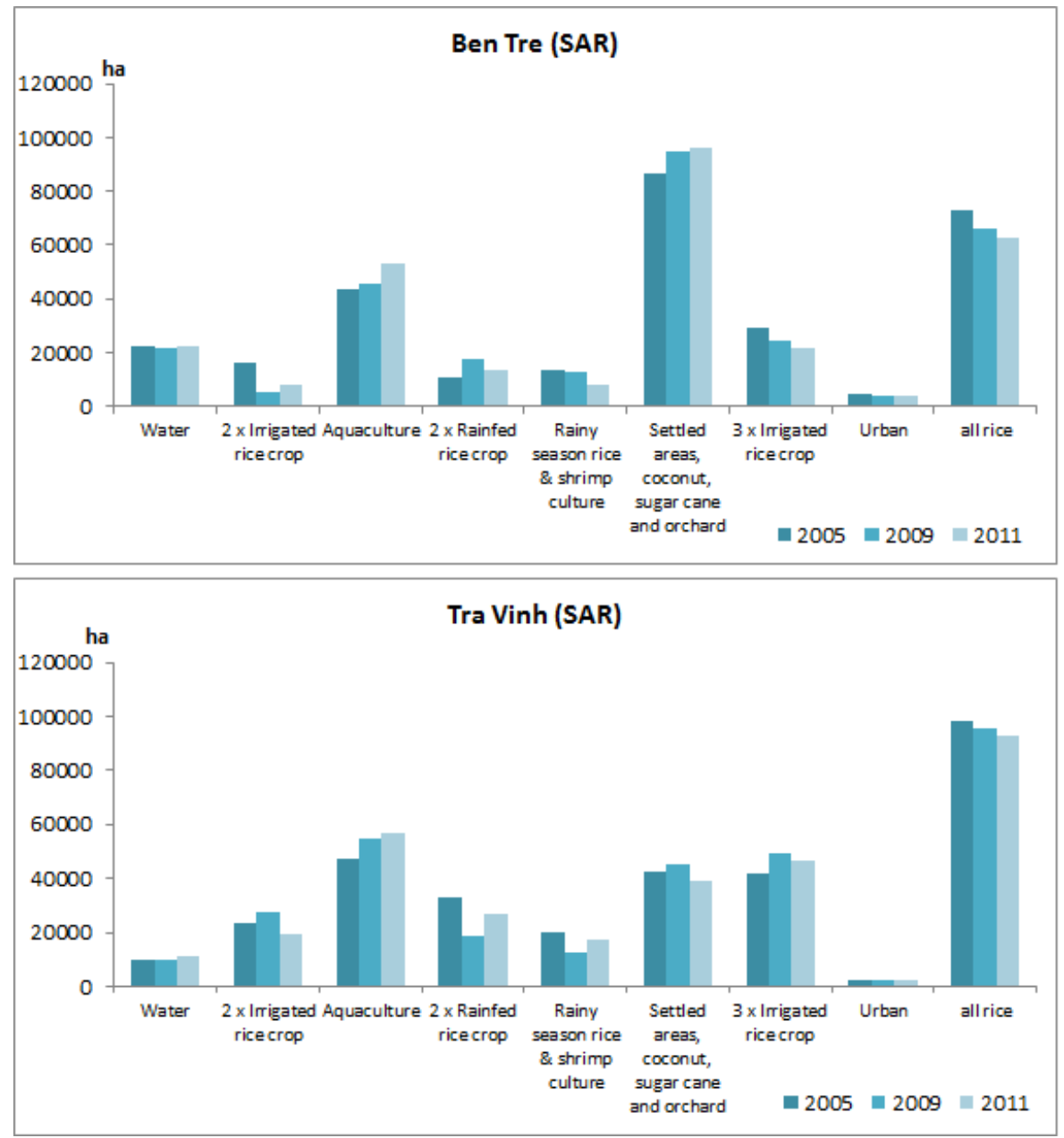

Figure 7. The overall land use in Tra Vinh province based on optical images for years 1979, 1987, 2002 and 2005.

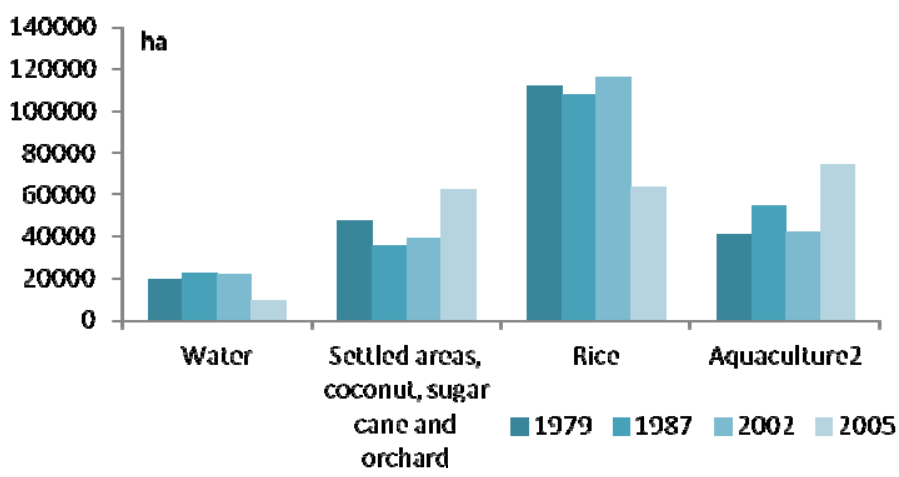


Figure 8. The SPOT results $(1987,1998,2005)$ for four districts in Tra Vinh.
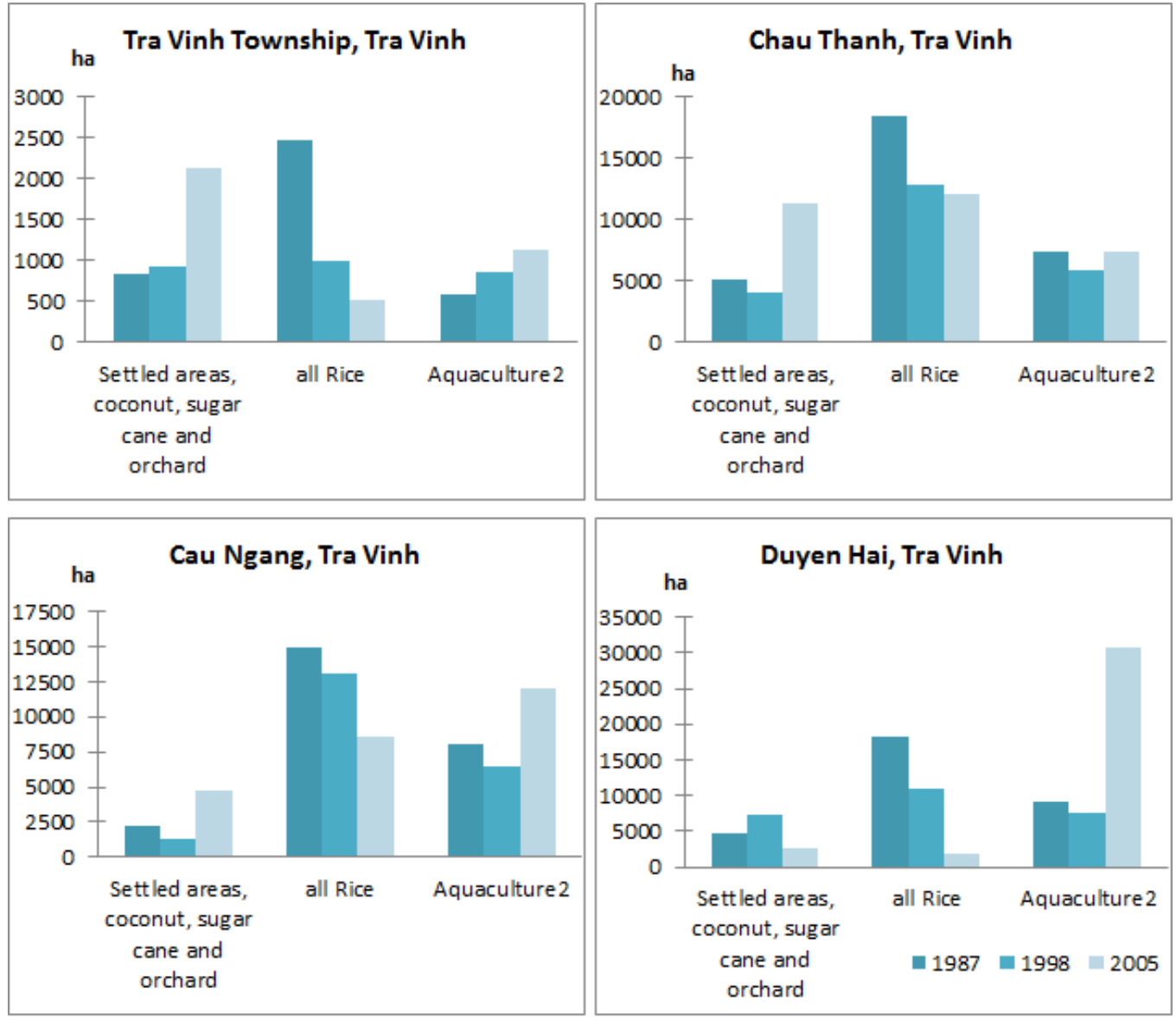

\subsection{Validation of the Land-Use Maps Using the Ground Truth Data}

The locations of the 75 field survey points within the study area are presented in Figure 1 . The validation of the land-use maps using ground truth data was performed to the 2005, 2009 and 2011 land-use maps derived from the ENVISAT ASAR data and to the land-use map created from the SPOT 2005 image. The accuracy of the ground truth is expected to decrease for data taken earlier than 2011/2012.

For validation, the pixel size of the land-use maps was downsampled from $75 \mathrm{~m}$ to $150 \mathrm{~m}$, which was the target pixel size of the final land-use maps. The following overall accuracies were achieved:

- $\quad$ Land-use map 2011 (SAR): 80.0\%

- Land-use map 2009 (SAR): 82.7\%

- $\quad$ Land-use map 2005 (SAR): 77.3\%

- Land-use map 2005 (SPOT 4): 88.2\%

The best overall accuracy with the SAR derived land-use maps was achieved in 2009, which also had the best availability of SAR data. The SPOT 2005 land-use map has a higher overall accuracy than SAR land-use maps, this is mainly explained with the fact that SPOT classification includes two classes less, as the "Rainy season rice and shrimp culture" class was merged with the "Aquaculture" class and the urban area was not classified. Due to the class division and the image extent only 68 ground truth points were used for the SPOT image. 
Table 6. Accuracy of the results.

\begin{tabular}{|c|c|c|c|c|c|}
\hline Class Name & $\begin{array}{c}\text { Reference } \\
\text { Totals } \\
\end{array}$ & $\begin{array}{c}\text { Classified } \\
\text { Totals } \\
\end{array}$ & $\begin{array}{l}\text { Number } \\
\text { Correct }\end{array}$ & $\begin{array}{c}\text { Producer's } \\
\text { Accuracy }\end{array}$ & $\begin{array}{c}\text { User's } \\
\text { Accuracy } \\
\end{array}$ \\
\hline \multicolumn{6}{|l|}{ ENVISAT 2005} \\
\hline Water & 4 & 7 & 4 & $100.00 \%$ & $57.14 \%$ \\
\hline All rice & 24 & 29 & 23 & $95.83 \%$ & $79.31 \%$ \\
\hline Aquaculture & 20 & 22 & 15 & $75.00 \%$ & $68.18 \%$ \\
\hline Rainy season rice and shrimp culture & 4 & 1 & 0 & $0.00 \%$ & $0.00 \%$ \\
\hline Settled areas, coconut, sugar cane and orchard & 19 & 12 & 12 & $63.16 \%$ & $100.00 \%$ \\
\hline Urban & 4 & 4 & 4 & $100.00 \%$ & $100.00 \%$ \\
\hline 2005 Totals & 75 & 75 & 60 & & \\
\hline \multicolumn{6}{|l|}{ ENVISAT 2009} \\
\hline Water & 4 & 6 & 4 & $100.00 \%$ & $66.67 \%$ \\
\hline All rice & 24 & 30 & 24 & $100.00 \%$ & $80.00 \%$ \\
\hline Aquaculture & 20 & 18 & 14 & $70.00 \%$ & $77.78 \%$ \\
\hline Rainy season rice and shrimp culture & 4 & 3 & 2 & $50.00 \%$ & $66.67 \%$ \\
\hline Settled areas, coconut, sugar cane and orchard & 19 & 14 & 14 & $73.68 \%$ & $100.00 \%$ \\
\hline Urban & 4 & 4 & 4 & $100.00 \%$ & $100.00 \%$ \\
\hline 2009 Totals & 75 & 75 & 62 & & \\
\hline \multicolumn{6}{|l|}{ ENVISAT 2011} \\
\hline Water & 4 & 5 & 4 & $100.00 \%$ & $80.00 \%$ \\
\hline All rice & 24 & 24 & 21 & $87.50 \%$ & $87.50 \%$ \\
\hline Aquaculture & 20 & 25 & 17 & $85.00 \%$ & $68.00 \%$ \\
\hline Rainy season rice and shrimp culture & 4 & 2 & 0 & $0.00 \%$ & $0.00 \%$ \\
\hline Settled areas, coconut, sugar cane and orchard & 19 & 15 & 14 & $73.68 \%$ & $93.33 \%$ \\
\hline Urban & 4 & 4 & 4 & $100.00 \%$ & $100.00 \%$ \\
\hline 2011 Totals & 75 & 75 & 60 & & \\
\hline \multicolumn{6}{|l|}{ SPOT 2005} \\
\hline Water & 3 & 3 & 3 & $100.00 \%$ & $100.00 \%$ \\
\hline All rice & 24 & 19 & 19 & $79.17 \%$ & $100.00 \%$ \\
\hline Aquaculture2 & 23 & 26 & 22 & $95.65 \%$ & $84.62 \%$ \\
\hline Settled areas, coconut, sugar cane and orchard & 18 & 20 & 16 & $88.89 \%$ & $80.00 \%$ \\
\hline SPOT 2005 Totals & 68 & 68 & 60 & & \\
\hline
\end{tabular}

The ground truth data was collected in October 2013 while the oldest land-use maps validated were from the year 2005. However, based on the information received from the local people during the field survey campaign, most of the significant changes in the study area occurred before the year 2005. A summary of the classification accuracies is provided in Table 6. The producer's accuracy is the percentage of reference points correctly classified. The user's accuracy describes the probability that a classified pixel actually belongs to the class. "Rainy season shrimp and rice" was often classified into the "All rice" or "Aquaculture" class, and thus the accuracies are low. To correctly classify the class more frequent imaging throughout the year is required. Other classes were to some extend classified into the "Settled areas, sugarcane, coconut and orchard" class, and thus the producer's accuracy is lower than the user's accuracy. The class has a diversity of different land covers, and thus, confusion with other classes occurs. Rice paddies and water areas were in a few cases classified as "Aquaculture"; 
likely due to the similar land cover characteristics. Low user's accuracies for "Water" are likely caused by flooded areas or aquaculture paddies misclassified as water bodies.

The trends in rice land-use changes based on the optical data appear to be supported by the interviews with the local people. However, it should be emphasized that the accuracy of the optical results before 2005 is uncertain as the data are single date data and because the only reference data available before 2005 was the interviews. According to the interviews in 2005, three rice fields changed to aquaculture, one changed from rice to aquaculture in 1995 and a rice field changed to a banana plantation in 2008 .

The EO data appears to support the assumption that the rice cultivated areas have decreased during the time period under investigation. At the same time, the aquaculture and coconut/orchard acreages have increased in the area. The result is in line with the information acquired from the farmers during the field survey campaign. According to the land-use maps based on the SAR imagery, $c a$. 19,000 ha of rice has changed to other land use between 2005 and 2011. Biggest relative changes have taken place in the inland districts of Ben Tre and Tra Vinh provinces. Moreover, the results from the optical imagery (Landsat 1979 and SPOT 2005) indicated that the land-use changes in coastal areas have occurred before the year 2005, which also was verified by the farmers in the target area. Also, Binh et al. [19] reported rapid increase in shrimp acreage in Cai Nuoc (Ca Mau province) around the year 2000 when a government decision allowed farmers to transform coastal saline rice fields into shrimp farms.

It should be noted that supervised methods or more advanced image classification algorithms, such as object based techniques or methods designed for SAR, might have improved the classification accuracies for single datasets. However, here we have focused on change detection and the aim was not to develop classification algorithms. According to our experience, land-use classification results are always dependent on the class division, the quality of the possible training data, test data and the characteristics of the study area including the size of the land-use units in comparison to EO data resolution. Therefore, qualitative comparison to other studies is not straightforward and more attention should be paid on the general trends in the historical changes. In a study by Tong et al. [20], land cover was classified in Tra Vinh and Ca Mau provinces from SPOT imagery with an average accuracy nearing $80 \%$, similarly to our results. In Bouvet and le Toan's research [28], ENVISAT WSM temporal backscatter change was used to derive rice area and was compared to statistical planted area information for 13 provinces in the Mekong delta region, and an $\mathrm{R}^{2}$ value of 0.92 was reported. They estimated the rice acreage at different seasons, however here we used the number of crops per year, and thus the results are not directly comparable.

\section{Conclusions}

An EO dataset of SAR and optical images covering a time period of around 30 years was acquired from Vietnam's Mekong Delta region, to study the long term changes in land use, with a special emphasis on rice, other cultivated areas and aquaculture. Land-use maps were produced using automatic classification techniques and the resulting land-use maps were validated using the reference data collected in a field survey campaign. In classifications an overall accuracy of $77 \%-88 \%$ was achieved.

Using the comprehensive EO data set in this study, it can be concluded that wide-area and up-to-date information on land-use and its changes can be obtained, which cannot be obtained by other 
means such as field surveys. We also observed that, together with the field validation data, the few interviews of local farmers provided a valuable contribution to analyzing the reliability and the accuracy of the land-use classification data. Therefore, additional in-situ inquiries are worth considering in studies, where archived data from the past EO experiments is needed for analyzing the present situation or to complement a time series.

The results presented here were limited by the amount of EO data available in the satellite image archives. Even though we carefully collected all available EO data acquired during the last 30 years, there were some caps in the areal extent, and this concerns mainly the optical EO data due to the cloudiness in the Mekong Delta region. Since the radar wavelengths penetrate clouds, SAR data is preferred. In order to carry out land-use change mapping, it is vital that SAR EO data is collected systematically throughout the year from the target area. However, it should be noted that image acquisition dates should always be carefully planned in order to interpret, e.g., multi-year rice crops.

The spatial resolution of the results presented here are limited to the resolution of the satellite data available, some $150 \mathrm{~m}$ in Envisat ASAR. The modern high resolution SAR satellites, such as TerraSAR-X, COSMO-SkyMed, Radarsat-2, would give more detailed results on land use. However, the amount of data needed also increases due to the smaller image coverage, which also limits the total area under investigation. Therefore, the wide-swath mode of the SAR is expected to play an important role in wide-area land-use mapping. In the future, from the rice mapping point of view, the use of Sentinel-1 satellites of the European Space Agency appears to be a very promising approach, because they could allow continuous wide-area monitoring of areas such as the Mekong Delta region with a good temporal coverage. Up-to-date land-use maps from Vietnam are constantly needed to detect long-term trends in land-use and to be used as an aid in decision making when policies concerning farming actions are planned. This information can be produced from SAR satellite data, with the help of optical EO data.

\section{Acknowledgments}

This study was done in cooperation with Roshan Cooke from the United Nations International Fund for Agriculture and Torsten Bondo from the European Space Agency. The authors would like to thank the organizers of the field survey and the local authorities taking part in the survey. In addition, authors would like to thank the anonymous reviewers for their valuable comments. The study was funded by the ESA through the project "Earth Observation Information Services for United Nation International Fund for Agricultural Development, Annex E: Crop Type Monitoring in Vietnam”. The manuscript writing was partly funded by the Academy of Finland project: "Mobile hyperspectral laser remote sensing". The ENVISAT imagery was provided by ESA within the framework of Category-1 project 14134 "Crop type monitoring in Vietnam". The Landsat data were acquired from the data archives of the U.S. Geological Survey. The SPOT data were distributed by the Astrium Services/Spot Image S.A., France.

\section{Author Contributions}

Kirsi Karila wrote the majority of the paper. Kirsi Karila and Mika Karjalainen designed the methodology used in the study. Olli Nevalainen and Anssi Krooks processed the image data, 
collected the field data and wrote the Methods section of the paper. Kirsi Karila, Olli Nevalainen and Anssi Krooks interpreted the results. Sanna Kaasalainen contributed to the organization of the paper. Mika Karjalainen and Sanna Kaasalainen supervised the study and contributed to the Introduction and the Conclusions sections of the paper. All authors participated in the editing and revision of the manuscript.

\section{Conflicts of Interest}

The authors declare no conflict of interest.

\section{References and Notes}

1. Rice in Vietnam, The www Page of the International Rice Research Institute. Available online: http://irri.org/index.php?option=com_k2\&view=item\&id=8762:rice-in-vietnam\&lang=en (accessed on 25 November 2013).

2. Le Toan, T.; Ribbes, F.; Wang, L.; Floury, N.; Ding, K.; Kong, J.A.; Fujita, M.; Kurosu, T. Rice crop mapping and monitoring using ERS-1 data based on experiment and modeling results. IEEE Trans. Geosci. Remote Sens. 1997, 35, 41-56.

3. Brisco, B.; Brown, R.J. Multi-date SAR/TM synergism for crop classification in Western Canada. Photogramm. Eng. Remote Sens., 1995, 61, 1009-1014.

4. Hong, G.; Zhang, A.; Zhou, F.; Townley-Smith, L.; Brisco, B.; Olthof, I. Crop type identification potential of Radarsat-2 and MODIS images in prairie area. Can. J. Remote Sens. 2011, 37, 45-54.

5. Blaes, X.; Vanhalle, L.; Defourny, P. Efficiency of crop identification based on optical and SAR image time series. Remote Sens. Environ. 2005, 96, 352-365.

6. Michelson, D.; Liljeberg, M.; Pilesjö, P. Comparison of algorithms for classifying Swedish landcover using Landsat TM and ERS-1 SAR data. Remote Sens. Environ. 2000, 71, 1-15.

7. Ban, Y. Synergy of multitemporal ERS-1 SAR and LANDSAT TM data for classification of agricultural crops. Can. J. Remote Sens. 2003, 29, 518-526.

8. Brisco, B.; Brown, R.J.; Manore, M.J. Early season crop discrimination with combined SAR and TM data. Can. J. Remote Sens. 1989, 15, 44-54.

9. Le Hegarat-Mascle, S.; Quesney, A.; Vidal-Madjar, D. Land cover discrimination from multitemporal ERS images and multispectral Landsat images: A study case in an agricultural area in France. Int. J. Remote Sens. 2000, 21, 435-456.

10. Schistad-Solberg, A.; Jain, A.; Taxt, T. Multisource classification of remotely sensed data: Fusion of Landsat TM and SAR Images. IEEE Trans. Geosci. Remote Sens. 1994, 32, 768-776.

11. Torbick, N.; Salas, W.; Xiao, X.; Ingraham, P.; Fearon, M.; Biradar, C.; Zhao, D.; Liu, Y.; Li, P.; Zhao Y. Integrating SAR and optical imagery for regional mapping of paddy rice attributes in the Poyang Lake Watershed, China. Can. J. Remote Sens. 2011, 37, 17-26.

12. McNairn, H.; Champagne, C.; Shang, J.; Holmstrom, D.A.; Reichert, G. Integration of optical and Synthetic Aperture Radar (SAR) imagery for delivering operational annual crop inventories. ISPRS J. Photogramm. Remote Sens. 2009, 64, 434-449. 
13. Erasmi, S.; Twele, A. Regional land cover mapping in the humid tropics using combined optical and SAR satellite data: A case study from Central Sulawesi, Indonesia. Int. J. Remote Sens. 2009, 30, 2465-2478.

14. Xiao, X.; Boles, S.; Frokling, S.; Li, C.; Badu, J.; Salas, W.; Moore, B. Mapping paddy rice agriculture in South and Southeast Asia using multi-temporal MODIS images. Remote Sens. Environ. 2006, 100, 95-113.

15. Leinenkugel, P.; Esch, T.; Künzer, C. Settlement detection and impervious surface estimation in the Mekong Delta using optical and SAR remote sensing data. Remote Sens. Environ. 2011, 115, 3007-3019.

16. Gebhardt, S.; Huth, J.; Lam Dao, N.; Roth, A.; Kuenzer, C. A comparison of TerraSAR-X Quadpol backscattering with RapidEye multispectral vegetation indices over rice fields in the Mekong Delta, Vietnam. Int. J. Remote Sens. 2012, 33, 7644-7661.

17. Kuenzer, C.; Knauer, K. Remote sensing of rice crop areas-A review. Int. J. Remote Sens. 2013, 34, 2101-2139.

18. Nguyen, T.T.H.; de Bie, C.A.J.M.; Ali, A.; Smaling E.M.A.; Chu, T.H. Mapping the irrigated rice cropping patterns of the Mekong Delta, Vietnam, through hyper-temporal SPOT NDVI image analysis. Int. J. Remote Sens. 2012, 33, 415-434.

19. Binh, T.N.K.D; Vromant, N.; Hung, T.H.; Hens, L.; Boon, E.K. Land cover changes between 1968 and 2003 in Cai Nuoc, Ca Mau Peninsula, Vietnam. Environ. Dev. Sustain. 2005, 7, 519-536.

20. Tong, P.H.S.; Auda, Y.; Populus, J.; Aizpuru, M.; Al Habshi, A.; Blasco, F. Assessment from space of mangroves evolution in the Mekong Delta, in relation with extensive shrimp-farming. Int. J. Remote Sens. 2004, 25, 4795-4812.

21. Sakamoto, T.; Phung, C.V.; Kotera, A.; Nguyen, K.D.; Yokozawa, M. Analysis of rapid expansion of inland aquaculture and triple rice-cropping areas in coastal area of the Vietnamese Mekong Delta using MODIS time-series imagery. Landsc. Urban Plan. 2009, 92, 34-46.

22. WISDOM-Project Homepage. Available online: http://wisdom.eoc.dlr.de/ (accessed on 28 February 2014).

23. Chakraborty, M.; Panigrahy, S.; Sharma, S.A. Discrimination of rice crop grown under different cultural practices using temporal ERS-1 SAR data. ISPRS J. Photogramm. Remote Sens. 1997, 52, 183-191.

24. Liew, S.C.; Kam, S.-P.; Tuong, T.-P.; Chen, P.; Minh, V.Q.; Lim, H. Application of multitemporal ERS-2 synthetic aperture radar in delineating rice cropping systems in the Mekong River Delta, Vietnam. IEEE Trans. Geosci. Remote Sens. 1998, 36, 1412-1419.

25. Karjalainen, M.; Kuittinen, R.; Junnikkala, V.; Karvonen, T.; Nguyen, M.H.; Ha, T.T.T. Rice Yield Estimation Using SAR Images, Meteorological Data and GIS. In Proceedings of the ERS-Envisat Symposium, Gothenburg, Sweden, 16-20 October 2000.

26. Inoue, Y.; Kurosu, T.; Maeno, H.; Uratsuka, S.; Kozu, T.; Dabrowska-Zielinska, K.; Qi, J. Season-long daily measurements of multifrequency ( $\mathrm{Ka}, \mathrm{Ku}, \mathrm{X}, \mathrm{C}$, and $\mathrm{L}$ ) and full-polarization backscatter signatures over paddy rice field and their relationship with biological variables. Remote Sens. Environ. 2002, 81, 194-204. 
27. Bouvet, A.; le Toan, T.; Lam-Dao, N. Monitoring of the rice cropping system in the Mekong Delta using ENVISAT/ASAR dual polarization data. IEEE Trans. Geosci. Remote Sens. 2009, 47, 517-526.

28. Bouvet, A.; le Toan, T. Use of ENVISAT/ASAR wide-swath data for timely rice fields mapping in the Mekong River Delta. Remote Sens. Environ. 2011, 115, 1090-1101.

29. Kuenzer, C.; Guo, H.; Huth, J.; Leinenkugel, P.; Li, X.; Dech, S. Flood mapping and flood dynamics of the Mekong Delta: ENVISAT-ASAR-WSM based time series analyses. Remote Sens. 2013, 5, 687-715.

30. Choudhury, I.; Chakraborty, M. Analysis of temporal SAR and optical data for rice mapping. J. Indian Soc. Remote Sens. 2004, 32, 373-385.

31. Mekong Delta Water Resources Assessment Studies. Available online: http://nguyenthanhmy.com/ courses/GOOD-Mekong-Water-Resource-2011.pdf (accessed on 15 November 2013).

32. Tran, T.X.M.; Nguyen, D.V. Salinity Intrusion Trend in River and Canal Systems and Some Prevention Methods in Ben Tre Province. In Proceedings of the Vietnam-Japan Estuary Workshop 2006, Hanoi, Vietnam, 22-24 August 2006.

33. Preston, N., Clayton, H., Eds.; Rice-Shrimp Farming in the Mekong Delta: Biophysical and Socioeconomic Issues; ACIAR Technical Reports No. 52e; Australian Centre for International Agricultural Research: Canberra, ACT, Australia, 2003.

34. The Official Site of Ben Tre Province. Available online: http:/www.english.bentre.gov.vn (accessed on 15 March 2013).

35. Vietnam Archive Map Collection. Scanned Topographic Maps. Available online: http://www.vietnam.ttu.edu/virtualarchive/redirects/maps-redirect.htm (accessed on 14 November 2013).

36. Park, J.-M.; Song, W.J.; Pearlman, W.A. Speckle filtering of SAR images based on adaptive windowing. IEEE Proc. Vis. Image Signal Process. 1999, 146, 191-197.

37. Frost, V.S.; Stiles, J.A.; Shanmugan, K.S.; Holtzman, J.C. A Model for radar images and its application to adaptive digital filtering of multiplicative noise. IEEE Trans. Pattern Anal. Mach. Intell. 1982, doi:10.1109/TPAMI.1982.4767223.

38. Lavreau, J. De-Hazing Landsat thematic mapper images. Photogramm. Eng. Remote Sens. 1991, $57,1297-1302$.

39. Tou, J.T.; Gonzalez. R.C. Pattern Recognition Principles; Addison-Wesley Publishing Company: Reading, MA, USA, 1974.

40. Engdahl, M.; Hyyppä, J. Land-cover classification using multitemporal ERS-1/2 InSAR data. IEEE Trans. Geosci. Remote Sens. 2003, 41, 1620-1628.

41. Xuan, V. Rice cultivation in the Mekong Delta-Present situation and potentials for increased production. South East Asian Stud. 1975, 13, 88-111.

(C) 2014 by the authors; licensee MDPI, Basel, Switzerland. This article is an open access article distributed under the terms and conditions of the Creative Commons Attribution license (http://creativecommons.org/licenses/by/3.0/). 